\title{
Amenūkal, Amenoūkal
}

Voir Agellid, Ahaggar, Amyar, Attici, Moussa ag Amastane, Tawsit, Temaẓlait, Ti n Hinān, Tobol (ețtebel).(amənukal, emnokal, aménokal ; pluriel : imenūkālen, emnokalan ; féminin : tame-nukalt, timenūkālin)

\section{Gast et S. Chaker}

\section{OpenEdition}

\section{Journals}

Édition électronique

URL : http://journals.openedition.org/encyclopedieberbere/2471

DOI : $10.4000 /$ encyclopedieberbere.2471

ISSN : 2262-7197

\section{Éditeur}

Peeters Publishers

\section{Édition imprimée}

Date de publication : 1 septembre 1986

Pagination : 581-589

ISBN : 2-85744-282-3

ISSN : $1015-7344$

\section{Référence électronique}

M. Gast et S. Chaker, «Amenūkal, Amenoūkal », Encyclopédie berbère [En ligne], 4 | 1986, document A189, mis en ligne le 01 décembre 2012, consulté le 13 octobre 2020. URL : http:// journals.openedition.org/encyclopedieberbere/2471 ; DOI : https://doi.org/10.4000/ encyclopedieberbere.2471

Ce document a été généré automatiquement le 13 octobre 2020

(c) Tous droits réservés 


\section{Amenūkal, Amenoūkal}

Voir Agellid, Ahaggar, Amyar, Attici, Moussa ag Amastane, Tawsit, Temaẓlait, Ti n Hinān, Tobol (ețtebel).(amənukal, emnokal, aménokal ; pluriel : imenūkālen, emnokalan ; féminin : tame-nukalt, timenūkālin)

\section{Gast et S. Chaker}

1 (amənukal, emnokal, aménokal; pluriel : imenūkālen, emnokalan; féminin : tame-nukalt, timenūkālin)

\section{Par M. Gast}

2 Ce mot désigne le plus communément en pays touareg le ou un chef suprême, un chef puissant. Mais les variations de ses connotations au masculin, au féminin et féminin pluriel sont nombreuses. Nous donnerons d'abord les différents sens de ce mot pour ensuite définir sommairement les attributions, la fonction et le rôle de l'« amenoûkal » chez les Touaregs.

1. Chef suprême, d'une fédération ou confédération de tawsit (tribus), héritier en voie utérine du pouvoir de commandement ou atţ̦bəl (tobol= tambour), symbolisé par l'attribution d'un gros tambour battu par les artisans pour réunir les guerriers ou le peuple auprès de son chef.

Le prestige moral, la puissance, la richesse et la culture proprement touarègue s'attachent au titre d'« amenoûkal ». Un chef non touareg, non prestigieux ou qui ne représente pas avec ses tawsit une organisation socio-politique reconnue comme " touarègue » ne sera jamais désigné par le nom officiel, consacré d'« amenoûkal ».

4 2. En revanche, par extension, on peut situer le rang d'un haut personnage (roi, empereur, sultan, chef indépendant, chef politique) puissant ou non, quelle que soit sa nation ou sa religion, en le désignant comme "amenoûkal ", faute d'autre vocable spécifique à la langue touarègue. De Foucauld ajoute même : «dans le sens de "chef puissant", se dit des chefs européens d'un grade un peu élevé, civils ou militaires; dans ce sens, sert à désigner les officiers de tous les grades des armées européennes et les administrateurs civils européens.»(Dict. III, p. 1213-1214). Nous avons assez peu 
constaté ce genre d'emploi en Ahaggar de 1950 à 1981. Nous avons entendu l'amenoûkal de l'Ahaggar désigner en 1951 le président de la République française par le terme assez rare de ulilyan, qui d'après nos interlocuteurs définit un chef encore plus important que l'amenoûkal touareg.

5 3. Amenoûkal peut aussi désigner au sens figuré un homme quelconque, même esclave, «soit comme expression laudative, pour exprimer que par ses qualités morales, intellectuelles, ou physiques, cet homme est comme un roi, soit pour signifier que, comme aisance ou richesse, cet homme est comme un roi, c'est-à-dire qu'il a largement le nécessaire si c'est un esclave, qu'il est à l'aise ou riche si c'est un homme libre » (Ch. de Foucauld, Dictionnaire touareg-français, t. II, p. 1214).

6 4. Les hommes de la famille des Imenanes, clan qui commandait l'Ahaggar, l'Ajjer et une partie de l'Aïr et de l'Adrar des Iforas jusqu'à la moitié du xviie siècle (et que l'histoire orale désigne comme d'essence maraboutique en provenance du Sud marocain), sont tous appelés "amenoûkal» ou "sultan" (essulțan) mot arabe employé aussi en touareg. A ce clan qui, pensons-nous, a bénéficié d'une "double filiation unilinéaire " (religieuse maraboutique par les hommes et touarègue noble par les femmes, voir $\mathrm{M}$. Gast, 1976, p. 48), s'attachait un très grand prestige. Tout individu, homme ou femme, même s'il n'en avait pas la fonction était désigné par l'appellation d'amenūkal, ou de tamenūkalt, de sa naissance à sa mort, qui signifie dans ce sens "prince » ou "princesse ", comme le signale de Foucauld: "ils sont comme des titres nobiliaires appartenant, par droit de naissance, à tout membre de leur famille, sans compter aucune autorité » (p. 1214).

7 Lorsqu'en 1875 les derniers Imenanes déchus de tout pouvoir sont malmenés et humiliés par les Ouraghen, clan régnant à l'époque sur le pays Ajjer, les femmes Imenanes timenūkalin viennent implorer le soutien des Kel-Ahaggar. Le scandale est alors grand chez ces derniers qui accusent les Ouraghen d'un manquement grave aux règles de "chevalerie touarègue ». Une guerre fratricide de plusieurs années (voir Benhazera, 1908, p. 117 et sq.) va décimer la jeunesse touarègue et créer un fossé irrémédiable, encore important aujourd'hui, entre les deux communautés. C'est dire, sur le plan culturel et politique, la forte charge affective et symbolique que comportent dans certains cas les titres d'amenūkal et de tamenūkalt.

8 5. Le père de Foucauld signale enfin le nom d'un fusil de guerre qui, à la fin du XIX siècle, fut importé du Maroc en Ahaggar: wa $\mathrm{n}$ amenūkal (celui de l'amenūkal). Amenūkal désignant ici le Sultan de Maroc.

Ce nom d'origine berbère ancienne a donné lieu à un certain nombre d'interprétations erronées et fantaisistes soit de la part des Européens, soit de la part des Maghrébins eux-mêmes. Une croyance^ ${ }^{\wedge}$ persistante tend à donner à ce terme la signification de «possesseur ou propriétaire des terres ». C'est ce qu'a écrit par exemple H. Lhote dans son livre Les Touaregs du Hoggar (1955, p. 189). L'amenūkal étant le garant de l'intégrité du territoire commun à son tobol, on a tendance à lui appliquer le rôle d'un chef de patrimoine, de cette terre collective (habous ou waqf comme chez les Arabes) qui représente une propriété indivise. Par ailleurs en Ahaggar même, certains Touaregs arabisés vont jusqu'à répercuter l'explication suivante : l'amenūkal est le chef de la terre comme son nom l'indique, amin akal. Amin, nom arabe, qui désigne une personne de confiance, un ministre, etc. ; akal, étant le mot berbère désignant le pays, la terre collective. 

faire glisser la fonction et le titre d'amenūkal dans les cadres de la culture et de la langue arabe, en gommant sa spécificité, sa « différence » qui ne correspond à aucun concept ou structure du monde arabo-islamique.

11 L'on comprendra mieux ce qu'est un "amenoûkal » en analysant sa fonction, ses attributions et le rôle qu'il a dans l'unité politique, territoriale et culturelle que forme son tobol. L'exemple d'amenoûkal le mieux connu et le mieux étudié est celui des Touaregs de l'Ahaggar dont le dernier héritier, douzième de sa lignée, s'est éteint en 1975 à Tamanrasset.

Dans un territoire commun (ațtabel ou tobol) totalement inaliénable, un certain nombre de groupes de parenté (tawsit ou tawšit) vivent autour d'un chef suprême: l'amenoûkal.

13 En Ahaggar les tawsit se définissent en deux catégories: celle des suzerains (Ihaggaren), celle des tributaires (Imyad ou Kel-Ulli). Aucun lien d'alliance matrimoniale n'unissait ces deux classes. Leurs relations étaient d'ordre politique et économique. Les artisans inaden, en très petit nombre, formaient une caste aux attributions spécifiques: endogames, chargés de l'art du feu, de la conservation de certains rites et traditions en relations de clientèle avec les uns et les autres. Les esclaves demeuraient la main d'œuvre servile obtenue grâce aux guerres de pillage effectuées en zone sahélienne.

Le chef suprême est choisi parmi les héritiers mâles dans un clan de suzerains reconnu comme celui pourvu du commandement. En Ahaggar c'est le clan des Kel-үela qui jouit de cet avantage en vertu du pouvoir de son ascendant féminin Tin-Hinan, « mère » des suzerains.

ajorité des clans dans tout le monde touareg, suzerains ou tributaires, fait toujours référence à un ancêtre féminin et les aristocrates, possesseurs du droit d'accès au commandement, à quelques exceptions près, justifient presque toujours leur pouvoir par référence à une femme, une mère commune. Nous considérons que cette spécificité de l'idéologie du pouvoir chez les Touaregs a fortement contribué à sauvegarder leurs structures socio-politiques et les a protégés contre l'accaparement de pouvoir par les étrangers (en particulier par les arabophones faisant référence à leur patrilinéarité).

En Ahaggar, l'amenūkal est élu par tous les amyar (ou chefs de clan) représentant les clans tributaires et par les aristocrates chefs de famille. Il est choisi parmi plusieurs prétendants, héritiers du droit au commandement en ligne utérine et dont on connaît parfaitement la généalogie jusqu'à quatre ou cinq générations (parfois jusqu'à plus de dix comme chez les Kel-Kummer de l'Azawag). Il est choisi en fonction de son caractère, de ses qualités morales et physiques, de son courage et de sa générosité, et quelques fois aussi de son âge (cependant, Moussa ag Amastan a accédé au titre d'amenoûkal à moins de trente ans, dans des conditions politiques exceptionnelles, il est vrai). Une confiance réciproque et de solides liens affectifs doivent s'établir entre l'amenoûkal et son peuple, faute de quoi il ne pourra avoir d'autorité réelle, car il ne dispose d'aucun appareil de coercition pour faire appliquer sa justice et ses ordres. Il ne peut s'appuyer que sur le consensus. C'est aussi la raison pour laquelle il est "révocable " à tout moment par la volonté de son peuple (les exemples ne manquent pas).

Les attributions liées à la fonction d'amenūkal sont les suivantes :

- il reçoit annuellement comme impôt et marque d'allégeance un produit en nature sur 
les troupeaux, les marchandises issues du commerce caravanier, payé par les utérins chez les tributaires. Ce tribut, la tiwsé représente son principal revenu,

- une redevance sur les jardins cultivés sur le territoire de l’atţ̦bel (2,5 kg d'orge ou de blé par « jardin » ou unité d'arrosage),

- des droits de passage sur les caravanes traversant son territoire,

- des parts de butin sur les rezzous des Kel-Ulli vainqueurs, mais pas sur le butin des aristocrates, ses pairs,

- des redevances accordées par des tawsit extérieures à son əțțəbel mais qui sollicitent sa protection. Cette redevance ou "part spéciale » est appelée temazlayt comme celle qu'accordent certains matrilignages de Kel-Ulli à un suzerain de leur atțəbel pour bénéficier de sa protection contre les pillages des autres suzerains.

L'amenūkal jouit aussi des biens en déshérence qui forment pour les troupeaux un «trésor public " vivant, inaliénable et dont il a l'usufruit. Il peut aussi en faire bénéficier les pauvres qui le sollicitent (prêts de chamelles en lactation, chèvres ou vaches s'il en existe).

19 A titre personnel il reçoit :

- les profits accordés à son propre lignage en " part spéciale », moyennant sa protection sur les matrilignages payant cette temaẓlayt ;

- de nombreux cadeaux complémentaires aux revenus ci-dessus, offerts par des étrangers, des Kel-Ulli ou autres personnes venues solliciter son aide ou ses bonnes grâces pour toute sortes de raisons.

L'amenūkal rend la justice. Il arbitre les différends à tous les niveaux. Il joue en quelque sorte le rôle du šayx des tribus arabes. Il fait souvent la charité aux pauvres, aux nécessiteux de toute classe qui le sollicitent (dons de céréales, beurre, fromages, prêt de femelles en lactation surtout). Il intervient dans les associations économiques concernant les lignages suzerains et les lignages tributaires (temazlayt) pour permettre la survie des segments lignagers des suzerains exclus du droit d'accès au commandement. Car les Kel-Ulli «nourrissent » au sens propre les suzerains. Il décrète la guerre collective avec l'accord et l'appui des autres suzerains en cas de menace, de besoin, ou pour défendre l'honneur et la cohésion morale de son groupe.

21 L'amenūkal est donc le point de convergence de la vie du tobol touareg, la grille de sécurité et la référence qui permet le bon fonctionnement de la vie collective, qu'elle soit politique, économique, sociale ou morale. Cependant, ses pouvoirs ne peuvent s'exercer qu'en fonction de la cohésion morale de son əțţbel ou tobol et de la force de l'idéologie qui sous-tend cet ensemble. Les cas d'indiscipline de ses pairs sont nombreux ; il n'a aucun moyen direct, personnel, pour faire prévaloir son autorité sur ces derniers, sinon celui de faire jouer les uns contre les autres, en invoquant les règles coutumières dont il est le principal garant, en faisant référence à l'idéologie touarègue toujours très importante comme charge affective et dynamique chez ces nomades. Mais l'ascendant moral qu'il aura acquis par son courage, sa résistance physique au combat, ses qualités intellectuelles, politiques et surtout sa générosité, seront par dessus tout des facteurs prépondérants. Moussa ag Amastan fut dans l'esprit des Kel-Ahaggar le modèle le plus accompli de ce type de chef.

D'autres termes désignent en pays touareg le chef suprême ou un chef quelconque. Une analyse comparée de tous ces termes serait possible. Celle-ci pourrait montrer les variations des structures d'autorité politique et sociale, et le niveau de prestige consacré à chacun d'eux. Par ailleurs, nous constatons que les termes désignant le chef 
subissent l'influence des langues et des ethnies avoisinant ou cohabitant avec les Touaregs. Nous citerons amyar, askia, anastafidet, tambeh (tamboli), baba-n-serki (en hausa), moro-naba, sultan.

Chez les «Touaregs du nord » bien qu'il y eut trois əțțəbel (Kel-үela, Taïtoq, Téğehé Mellet) en principe indépendants économiquement et politiquement les uns des autres, seul le chef des Kel-үela, nettement plus puissant que les autres, a toujours porté le titre d'amenūkal. Les autres n'ont jamais été nommés que par le terme de amyar. Il n'y a pas de terme spécifique comme c'est le cas aussi en arabe, intermédiaire entre amenūkal (ou rays) et amүar (ou šayx) quand ce dernier est amүar d'amyar (ou šayx de šayx : šayx elmašayx).

Autrement dit, il ne peut y avoir de petit amenūkal dans l'esprit de la langue touarègue.

Liste des amenūkal de l'Ahaggar connus par les traditions orales et l'histoire.

1. Salah : milieu du XVIII ${ }^{\mathrm{e}}$ siècle.

2. Moxammed el-Xeir ag Salah : deuxième moitié du XviII ${ }^{e}$ siècle.

3. Sidi ag Moxammed el-Xeir : fin du XvIII ${ }^{e}$ siècle.

4. Yunès ag Sidi : vers $1790 \ldots 1820$ (?).

5. Ag Mama ag Sidi : $1820 \ldots 1860$ (?).

6. El-Hağ Axmed ag El-Hağ El-Bekri : 1860-1877.

7. Ahitayel ag Moxammed-Biska : 1877-1900.

8. Moxammed ag ūrzīg :

8 bis.Atisi ag Amellal : 1900-1904

9. Mūsa ag Amastan : 1905-1921.

10. Axamuk ag Ihemma : 1921-1941.

11. Meslay ag Amayas : 1941-1950.

12. Bey ag Axamuk : 1950-1975.

\section{Amenūkal/M N K D (.) (S. Chaker)}

25 «Imperator, roi, chef suprême... » [étymologie].

La question de l'origine et de la formation du couple (libyque) M N K D (.)/(touareg) amenūkal a été abordée par de nombreux auteurs, sans qu'aucune thèse ne fasse le consensus. Il paraît cependant possible de clarifier quelque peu le débat en cernant et en sériant les problèmes et, surtout, en respectant les précautions de méthode qui s'imposent en matière d'étymologie. Des hypothèses explicatives sérieuses - parce que compatibles avec les contraintes structurales et les données lexico-sémantiques de la langue berbère - peuvent alors être formulées.

Le couple MNKD/Amenūkal suscite en fait deux interrogations principales: $1^{\circ}$ S'agit-il d'un seul et même terme? (MNKD étant la forme antique, amenūkal, la forme moderne touarègue du même mot.)

La thèse de l'identité-continuité MNKD/amenūkal a longtemps prévalu. Proposée par Lévi Della Vida (1935), elle a été généralement admise tant la forme et le sens semblaient proches. Elle a été mise en doute par Rössler (1964), puis Garbini (1968) et Galand (1968). Nous avons nous-mêmes émis de sérieuses réserves à son propos (Chaker, 1973). La question doit être examinée sous ces deux faces, signifié et signifiant.

28 - Du point de vue du sens, la thèse de l'identité MNKD/amenūkal est, à première vue, très forte. Elle tient même de l'évidence. Le MNKD libyque correspond au latin Imperator. Le 
terme touareg amenūkal désigne "le roi, le chef suprême, le sultan, un chef très puissant... » (Foucauld, 1951, III, p. 1213 et Gast, supra). Il y a donc quasi identité sémantique.

Avec toutes les précautions qu'impose une documentation très limitée, il semble que le titre libyque ait été moins précis que l'actuel amenūkal touareg. De par sa fréquence et le détail de ses emplois dans les inscriptions libyque (Chaker, 1986), MNKD semble désigner un personnage important, mais pas nécessairement le détenteur d'un pouvoir suprême.

Si l'on admet la suggestion de G. Camps (1985) d'y voir plutôt une fonction militaire (par opposition au GLD-agellid, civil ou religieux), on peut retenir l'interprétation: "officier supérieur", "détenteur d'un commandement militaire élevé», "chef d'armée »... J'ai proposé (1986) d'y voir l'équivalent du dux latin ou du Herzog germanique (dans sa signification primitive). On verra que l'étymologie que nous en proposons tendrait à confirmer cette hypothèse.

31 - Sur le plan de la forme, en revanche, le rapprochement MNKD/amenūkal est beaucoup plus hasardeux. Il est même assez improbable.

32 La dernière consonne de la séquence libyque /-D/ correspond à /1/ en touareg. Une évolution: apico-dentale /d/ > apicale latérale /l/ n'aurait rien d'impossible, ni en phonétique générale (pour laquelle le processus ne serait pas extraordinaire), ni même en phonétique berbère. Des tendances à la confusion entre / $/$ et $/ d /$ existent dans certains parlers berbères actuels où la liquide /l/ peut être traitée en dentale palatalisée /d'/ ou en affriquée /dž/. On relève même quelques cas isolés d'alternance libre entre /l/ et /d/ pour un même mot : le kabyle commun ayla " propriété » connaît une variante littéraire archaïque (proverbes) ayda. Mais il s'agit là de phénomènes rares et sporadiques : fondamentalement $/ \mathrm{d} /$ et $/ \mathrm{l} /$ sont deux phonèmes bien distincts en berbère où ils assument de nombreuses oppositions lexicales.

Comme nous n'avons pas les maillons intermédiaires entre le libyque et le touareg et que amenūkal n'existe qu'en touareg (aucune forme approchante n'a jamais été signalée dans les autres dialectes berbères), pour pouvoir considérer comme sérieuse l'hypothèse /-D/ > /-1/ dans le couple étudié, il faudrait :

Ou bien montrer que la correspondance libyque /-D/ berbère /-1/ n'est pas isolée et se rencontre dans d'autres séquences. On établirait ainsi la régularité d'une évolution phonétique. Telle n'est pas le cas puisqu'un terme libyque comme GLD (« roi ») conserve, dans tous les parlers modernes, sa finale ancienne /-D/ (> agellid...).

Ou bien pouvoir établir une correspondance régulière touareg /-1/ - autre(s) dialecte(s) berbère(s) /- $d /$, ce qui laisserait ouverte l'hypothèse d'une évolution spécifique /-D/ libyque $>/-1 /$ touareg. Or, les données actuelles ne permettent pas de poser une telle correspondance phonétique entre le touareg et un quelconque autre dialecte berbère.

Pour ce qui est du signifiant, il ressort donc que l'évolution MNKD > amenūkal, sans être tout à fait exclue, est assez improbable. Dans l'état actuel des connaissances, aucun indice sérieux ne permet de l'étayer.

En conclusion, tant qu'aucun élément de démonstration contraire n'aura été apporté, MNKD et amenūkal doivent, malgré leur quasi-identité sémantique, être considérés comme des formes étrangères l'une à l'autre.

$2^{\circ}$ Comment ces mots sont-ils formés? 
L'identité des deux formes étant des plus hypothétiques, il faut évidemment admettre la possibilité de formations distinctes. Pour les deux termes, on restera dans le cadre d'une étymologie autochtone berbère, mais un emprunt à une langue étrangère n'est pas à exclure.

\section{- MNKD(.) [libyque] :}

La dernière lettre libyque, de valeur incertaine, n'étant pas toujours présente (RIL, 83, $84,124,343,440,713,716,1076,1079 \ldots$...ne la comportent pas), c'est bien une forme MNKD qui doit être retenue. Le dernier caractère alternant représente certainement un suffixe grammatical (possessif ou personnel, déictique...), extérieur au mot lui-même.

41 Dans le cadre du système berbère de formation du mot, MNKD s'analysent immédiatement comme un "Nom d'agent » dérivé (par préfixe $m$-) d'un radical. Or, le touareg possède un verbe enked (forme déjà évoquée par O. Rössler, 1964, p. 126) qui a les significations suivantes :

42 Ahaggar : «aller au devant de / aller à la rencontre de / empêcher d'arriver jusqu'à soi / tenir loin de soi / se préserver de» (Foucauld, 1951, III, p. 1386); Aïr/Iwellemmeden : " aller au devant de / faire attention / se méfier / être averti de "

(Alojay, 1980, p. 146), ou: "aller au devant de / prévenir (par des mesures préventives) »(Prasse, 1970, p. 130).

Le verbe est également attesté au Mzab avec un sens compatible : « examiner, regarder avec attention »(Delheure, 1984, p. 136).

Le nom d'agent issu des formes verbales actuelles (= amankad), signifie donc: Touareg: «celui qui va au devant de / fait attention à / se méfie de / se préserve de / tient à distance / prévient, prend des mesures préventives... » et, Mzab: «celui qui examine avec soin ».

A partir de la constellation sémantique actuelle, on peut poser l'hypothèse d'un signifié primitif : "prendre garde / prendre des précautions / faire attention... », qui autorise aussi bien une évolution vers les différents sens touaregs que vers la signification mozabite.

Le MNKD de l'Antiquité libyque a pu être le "responsable de la sécurité publique ", c'est-à-dire le chef militaire chargé de «tenir à distance l'ennemi, d'assurer la protection du groupe ou du royaume ».

Cette explication est à la fois simple et adéquate puisqu'elle couvre bien tous les usages $\mathrm{du}$ terme MNKD. Sans doute relativement imprécis à l'origine (= «Responsable militaire », "Chef de guerre »), MNKD a dû avoir tendance, sous l'influence des usages latins, à prendre l'acception plus précise de Imperator. Un processus tout à fait parallèle de spécialisation a pu être décelé pour le titre GLD (Chaker, 1985, p. 248).

Bien sûr, en matière d'étymologie, la prudence est toujours de rigueur et d'autres analyses sont possibles (comme celles développées par Garbini dans son article de 1968), mais elles reposent généralement sur une cascade d'hypothèses indémontrables et paraissent à la fois complexes et aléatoires.

50 Signalons enfin qu'Alvarez Delgado (1964) a établi un parallèle, très problématique à mon sens, entre le libyque MNKD et un titre guanche Mencey/Mensey, connu par les transcriptions espagnoles du XVII ${ }^{\mathrm{e}}$ siècle, qui signifiait également « roi » ou «chef». 
Récemment, T. Lewicki (1983) a rapproché ce couple (?) libyco-guanche MNKD/ Mencey d'un titre royal mandingue : Mansa. Ces deux pistes « exotiques » paraissent fragiles et plutôt aventurées: les matériaux guanches étant particulièrement obscurs et incertains, on ne peut guère espérer trouver quelque lumière de ce côté-là.

\section{- Amenūkal [touareg] :}

51 Les interprétations étymologiques de ce titre touareg sont nombreuses. Et, comme bien souvent en ce domaine, les motivations idéologiques et les à prioris de toute sortes conduisent aux distorsions les plus cocasses. Car mieux vaut rire de ces analyses "spontanées" (en réalité arabisante) qui décomposent amenūkal en < amin (arabe) + akal (berbère), « chef du territoire » ou < amin + leeuqal, « chef des notables »... Toutes ont en commun une méconnaissance totale (ou même une volonté d'occultation) de la langue berbère et tendent à soumettre des données berbères à des modèles linguistiques (et culturels) arabo-islamiques.

Tout essai d'explication de la formation de ce terme doit se placer dans le cadre des structures grammaticales de la langue berbère et, plus précisément, se plier aux contraintes des procédures de formation du mot dans cette langue.

Et de ce point de vue, on doit tout d'abord constater qu'amenūkal est une forme isolée au plan de la structure morphologique. On ne peut l'intégrer dans aucune série dérivationnelle bien établie. Pourtant, amenūkal est le correspondant nominal d'un verbe menukel "être chef suprême" (Foucauld, III, p. 1213), lui-même vraisemblablement à analyser comme une forme secondaire médio-passive à préfixe $m$ d'un radical NKL.

L'identification de ce radical verbal pose problème en touareg actuel : il y existe bien un verbe enkel, mais il signifie : "souiller, être sale » (Foucauld, III, p. 1374), ce qui paraît totalement incompatible avec le sens des dérivés amenūkal/menukel.

Dans le lexique touareg actuel, la base de dérivation de amenūkal/menukel paraît donc ne plus être attestée.

On peut cependant évoquer des verbes touaregs qui présentent des ressemblances de forme et une certaine compatibilité sémantique avec amenūkal auquel ils pourraient être apparentés (ils constitueraient ainsi des traces indirectes de l'ancienne base de dérivation):

- nekelwi : « être riche, à l'aise, avoir tout en abondance » (Foucauld, III, p. 1375) ;

- tunkel (qui est manifestement un dérivé à préfixe $t$ - d'un verbe $n k l)$ : « être dérobé aux regards » (Foucauld, IV, p. 1906). Mais il s'agit là de pures hypothèses et la relation morphologique et sémantique de ces verbes avec amenūkal resterait en tout état de cause obscure et isolée.

57 K. Prasse (1974, p. 296) a proposé de voir dans amenūkal un composé formé de * $m n+$ akal («pays»), le premier segment pouvant être la forme de singulier (non attestée) de Imenan, nom d'une ancienne tribu noble (suzerains de l'Ajjer-Ahaggar jusqu'au milieu du XVII ${ }^{\mathrm{e}}$ siècle), l'ensemble signifiant : " prince du pays ».

Cette thèse aurait pour elle de permettre de comprendre pourquoi amenūkal est inconnu en dehors du domaine touareg, puisque l'un de ses constituants appartiendrait en propre à la tradition historique des Touaregs (encore que l'on ne verrait pas très 
bien pourquoi le terme est employé par les Touaregs méridionaux qui échappaient au commandement des Imenan !).

Mais, du point de vue strict de la méthode linguistique, rien ne permet d'étayer, ni d'infirmer, dans l'état actuel des connaissances, une telle explication. Là encore, on entre dans le champs des hypothèses invérifiables, fondées sur les seules convictions et intuitions personnelles du chercheur.

En définitive, l'analyse étymologique confirme que, malgré la ressemblance troublante de sens et - à un moindre degré - de forme, il faut considérer MNKD et amenūkal comme deux termes étrangers l'un à l'autre.

61 MNKD admet une analyse très claire dans le cadre de la dérivation verbo-nominale berbère: ce peut être le Nom d'Agent d'un verbe pan-berbère NKD "prendre des précautions, faire attention, aller au devant...». Et cette étymologie convient parfaitement à ce que l'on peut percevoir de la nature de cette fonction libyque. amenūkal, qui n'est connu qu'en touareg, obéit à une formation qui demeure pour l'instant inexpliquée.

\section{BIBLIOGRAPHIE}

ALOJAY Gh. Lexique touareg-français, Copenhague, 1980.

ALVAREZ DELGADO J. « Inscriptiones libicas de Canarias, La Laguna-Tenerife, 1964 (notamment p. 243-245).

BENHAZERA M. Six mois chez les Touaregs du Ahaggar, A Jourdan, Alger, 1908, 234 pages.

BOURGEOT A. «Idéologie et appellations ethniques : l'exemple touareg. Analyse des catégories sociales ", Cahiers d'études afric, t. 48, 1972, p. 533-554.

CAMPS G. « Agellid, titre royal numide », Encyclopédie berbère, II, 1985, notice A92, p. 249-250.

CAMPS G. Massinissa ou les débuts de l'Histoire, Alger, 1961.

СНАВОТ J.B. Recueil des inscriptions libyques, Paris, 1940, (=RIL).

CHAKER S. « Libyque : épigraphie et linguistique », Encyclopédie berbère, à paraître.

CHAKER S. Textes en linguistique berbère..., Paris, C.N.R.S., 1984.

CHAKER S. « Agellid », Encyclopédie berbère, II, 1985, notice A92, p. 248-250.

CHAKER S. « A propos de la terminologie libyque des titres et fonctions », A.I.O.N. (Naples), 1986. DELHEURE J. Dictionnaire mozabite-français, Paris, S.E.L.A.F., 1984. Février J.G. « Que savons nous du libyque ? ", Revue africaine, 100, 1956, p. 263-273 (notamment p. 269-270).

FÉVRIER J.G. « La constitution municipale de Dougga à l'époque numide », Mélanges de Carthage..., Paris, Geuthner, 1964-65, p. 85-91.

FOUCAULD Ch. de, Dictionnaire touareg-français, Paris, 1950-51. 
GALAND L. Langue et littérature berbères. Vingt cinq ans d'études, Paris, C.N.R.S., 1979, p. 55. Garbini G. « Note libiche I et II », Studi Magrebini, 1, 2, 1968, (notamment, 2, § 6 : « Il significato della parola MNKD », p. 118-122).

GAST M. «Les Kel Rela : historique et essai d'analyse du groupe de commandement des Kel Ahaggar », Revue de l'Occident musulman et de la Méditerranée, t. 21, 1976, p. 47-63. « Pastoralisme nomade et pouvoir : la société traditionnelle des Kel Ahaggar ", Production pastorale et société, Maison des Sciences de l'homme et Cambridge press university press, 1979, p. 201-220.

LEVI della VIDA G. « Due iscrizioni neopuniche du Leptis Magna », Africa Italiana, VI, 1935.

LEWICKI T. «Sur le titre libyco-berbère des souverains Mandingues : Mansa », Études maghrébines et sahariennes, II, Varsovie, 1983, p. 32-41.

LHOTE H. Les Touaregs du Hoggar, Payot, Paris, 1955, 468 pages.

PRASSE K.G. Vocabulaire touareg. Tawellemmet de l'Est-français, Copenhague, 1970 (multigraphié).

PRASSE K.G. Manuel de grammaire touarègue, IV-V, N.O.M., Copenhague, 1974, p. 296-359.

RÖSSLER O. in H, Donner-W Röllig : Kanaanäische und Aramäische Inschriften, II (Kommentar), Wiesbaden, 1964, p. 126-127.

Voir Agellid, Ahaggar, Amyar, Attici, Moussa ag Amastane, Tawsit, Temaẓlait, Ti n Hinān, Tobol (ețțebel).

INDEX

Mots-clés : Histoire contemporaine, Linguistique, Pouvoir, Sahara, Sociologie 